\title{
Mapping of QTLs controlling seedling establishment using a direct seeding method in rice
}

\author{
Natsuko Iwata ${ }^{\dagger 1)}$, Hiroshi Shinada ${ }^{\dagger 2)}$, Hitoshi Kiuchi ${ }^{3)}$, Takashi Sato ${ }^{2)}$ and Kenji Fujino*1,4) \\ 1) Agricultural Research Institute, HOKUREN Federation of Agricultural Cooperatives, Higashi-5, Kita-15, Naganuma, Hokkaido \\ 069-1317, Japan \\ 2) Rice breeding group, Kamikawa Agriculture Experimental Station, Local Independent Administrative Agency Hokkaido Research \\ Organization, 1-5 Minami, Pippu, Hokkaido 078-0397, Japan \\ 3) Plant Genetic Resource Group, Central Agricultural Experiment Station, Local Independent Administrative Agency Hokkaido \\ Research Organization, Minami 363-2 Takinokawa, Takikawa, Hokkaido 073-0013, Japan \\ 4) Present address: National Agricultural Research Center for Hokkaido Region, Hitsujigaoka 1, Toyohira, Sapporo, Hokkaido 062-8555, \\ Japan
}

Low-temperature is one of the most common environmental stresses that affect plant growth and development and places a major limit on plant productivity. Tolerance to low-temperature is an important objective of rice breeding programs in temperate areas and at high altitudes in tropical and sub-tropical areas. Vigorous rice growth during the seedling stage at low-temperature is an important characteristic for stable seedling establishment (SES) in direct seeding methods, in which rice is sown directly into a flooded field. The aim of this study was to identify QTLs controlling SES using 3 mapping populations of backcrossed inbred lines (BILs) with Arroz Da Terra and Italica Livorno as the donor parents. SES was evaluated by a field evaluation system in this study, which is used in current rice breeding programs because of the complexity of environmental conditions in SES in rice cultivation in a paddy field. A total of 9 chromosomal regions for SES were identified, which explained $10.9 \%$ to $25.6 \%$ of total phenotypic variation. For all of QTLs except for $q S E S 5-2$, alleles from the donor parents increased phenotypic values. These QTLs should be useful for the improvement of SES in rice breeding programs in low-temperature regions.

Key Words: direct seeding method, Oryza sativa L., QTL, seedling establishment.

\section{Introduction}

Undesirable environmental conditions cause critical damage to plant growth and yield. These conditions could induce a wide range of plant responses, including altered gene expression and cellular metabolism, retarded growth, and reduced yield. Low-temperature is one of the most common environmental stresses that affect plant growth and development and places a major limit on plant productivity. The improvement of tolerance to low-temperature is an important objective in plant breeding programs.

Rice, Oryza sativa L., originated in a tropical region. Tolerance to low-temperature is an important objective of rice breeding programs in temperate areas, at high altitudes in tropical and sub-tropical areas, and in areas with a cold irrigation water supply (Nakagahra et al. 1997). Low-temperature causes the retardation of rice growth, from seed germination

Communicated by Qian Qian

Received June 25, 2010. Accepted September 27, 2010.

*Corresponding author (e-mail: kfujino@affrc.go.jp)

$\dagger$ These authors contributed equally to the work. to grain-filling stages. In the seedling (Peterson et al. 1978) and booting stages (Dai et al. 2004, Kuroki et al. 2007, Saito et al. 1995) in particular, rice plants are sensitive to lowtemperature, leading to the decreased rice yields.

Tolerance to low-temperature is a quantitative trait, which is controlled by many genes and affected by natural environmental conditions. Many QTLs have been identified as being involved in tolerance and response to low-temperature in rice, including seed germination stage (Fujino et al. 2004, Ji et al. 2009, Jiang et al. 2006, Miura et al. 2001, Zhang et al. 2005a), seedling stage (Andaya and Mackill 2003a, Baruah et al. 2009, Jiang et al. 2008, Lou et al. 2007, Zhang et al. 2005b), and booting stage (Andaya and Mackill 2003b, Dai et al. 2004, Kuroki et al. 2007, Saito et al. 1995, Suh et al. 2010, Takeuchi et al. 2001, Zhou et al. 2010). These QTLs could enhance tolerance to low-temperature in rice. However, it is not clear which is the most effective QTL for the improvement of tolerance to low-temperature for rice varieties in local regions, and which of them contribute to the variation of this trait in particular regions under local environmental conditions. For the application of QTLs identified using different genetic backgrounds under different natural 
environmental conditions in rice breeding programs in particular regions, it is necessary to validate them under local environmental conditions. Consequently, an important aim of rice breeding programs in local regions is to evaluate the response of a genotype under local environmental conditions in which the cultivars will be grown.

Hokkaido $\left(42-45^{\circ} \mathrm{N}\right.$ latitude) is the northernmost region of Japan and one of the northern-limits of rice cultivation in the world. Cool temperature during rice growth is a typical climatic condition in Hokkaido. In rice breeding programs in this region, tolerance to low-temperature during rice growth is the most important objective. Vigorous rice growth during the seedling stage under low-temperature is an important character for stable seedling establishment (SES) in direct seeding methods, in which rice is sown directly into flooded fields in Hokkaido. Seeds are sown in early May, when the temperature is still low for rice growth. Delayed and decreased emergence of rice seedlings from water because of low-temperature greatly increases seedling mortality (Peterson et al. 1978). Low SES leads to decreases in the number of plants grown in a paddy field, resulting in serious decreases in yield and grain quality.

For the development of new cultivars for direct seeding methods in local regions with low-temperature, introduction of stable SES is a priority in rice breeding programs. The phenotype of SES is involved in continuous growth from seed germination to early seedling growth. Furthermore, not only low-temperature but also other environmental factors, including soil conditions and anaerobic conditions due to flooding, cause a decrease in SES in rice production. However, it is unclear which environmental factors play major roles in mortality during SES.

The identification of the number and magnitude of gene effects could contribute to a better understanding of the genetic control of SES, facilitating the development of new cultivars with vigorous SES. The objective of this study was to identify QTLs controlling SES using 3 mapping populations of backcrossed inbred lines (BILs) with Arroz Da Terra and Italica Livorno as the donor parents. In this study, SES was evaluated by the field evaluation system used in the current rice breeding programs in Hokkaido. A total of 9 chromosomal regions for SES were identified. The relationships between the 9 QTLs identified in this study and known QTLs are discussed.

\section{Materials and Methods}

\section{Plant materials}

To evaluate varietal differences in SES, 24 rice varieties, including landrace and breeding types from Hokkaido, were used (Table 1). In addition, 3 varieties from Europe were used. These varieties were cultivated in a paddy field at Kamikawa Agricultural Experimental Station in 2009 (Pippu, Hokkaido, Japan).

Table 1. Variations of emergence of seedlings in soil (ESS) and emergence of seedlings from water (ESW) among rice varieties from Hokkaido

\begin{tabular}{|c|c|c|c|c|c|c|c|}
\hline \multirow{2}{*}{ Variety } & \multirow{2}{*}{ Origin } & \multirow{2}{*}{ Breeding } & \multicolumn{2}{|c|}{ ESS $(\%)$} & \multicolumn{2}{|c|}{ ESW (\%) } & \multirow{2}{*}{ Group } \\
\hline & & & NG & G & NG & G & \\
\hline Arroz Da Terra & Portugal & - & $84.7 \pm 3.1$ & $89.3 \pm 4.6$ & $72.0 \pm 4.0$ & $83.3 \pm 4.2$ & I \\
\hline Dunguang Shali & Hungary & - & $77.3 \pm 4.2$ & $87.3 \pm 1.2$ & $70.0 \pm 9.2$ & $82.7 \pm 4.2$ & I \\
\hline Italica Livorno & Italy & - & $66.0 \pm 2.0$ & $64.0 \pm 5.3$ & $60.7 \pm 3.1$ & $60.0 \pm 7.2$ & I \\
\hline Akage & Hokkaido & Landrace & $60.7 \pm 8.1$ & $61.3 \pm 5.0$ & $54.0 \pm 8.0$ & $52.7 \pm 8.1$ & II \\
\hline Fukoku & Hokkaido & 1935 & $56.7 \pm 1.2$ & $76.0 \pm 6.9$ & $50.0 \pm 5.3$ & $63.3 \pm 12.2$ & II \\
\hline Iburiwase & Hokkaido & Landrace & $52.0 \pm 2.0$ & $55.3 \pm 5.0$ & $46.0 \pm 2.0$ & $55.3 \pm 6.1$ & II \\
\hline Kitaibuki & Hokkaido & 1993 & $66.7 \pm 7.0$ & $74.7 \pm 7.6$ & $46.0 \pm 2.0$ & $66.0 \pm 4.0$ & II \\
\hline Tannemochi & Hokkaido & 1983 & $63.3 \pm 9.0$ & $70.0 \pm 7.2$ & $42.7 \pm 12.1$ & $48.0 \pm 5.3$ & III \\
\hline Chinkobouzu & Hokkaido & 1924 & $62.7 \pm 3.1$ & $66.7 \pm 4.2$ & $42.0 \pm 5.3$ & $58.7 \pm 6.1$ & III \\
\hline Daichinohoshi & Hokkaido & 2003 & $48.0 \pm 7.2$ & $56.0 \pm 14.0$ & $40.0 \pm 7.2$ & $54.0 \pm 8.7$ & III \\
\hline Yukimaru & Hokkaido & 1993 & $69.3 \pm 15.0$ & $83.3 \pm 7.6$ & $39.3 \pm 13.6$ & $66.0 \pm 10.6$ & III \\
\hline Kirara397 & Hokkaido & 1988 & $55.3 \pm 5.0$ & $58.7 \pm 4.2$ & $39.3 \pm 4.2$ & $47.3 \pm 4.6$ & III \\
\hline Hoshinoyume & Hokkaido & 1996 & $44.0 \pm 5.3$ & $70.0 \pm 6.9$ & $35.3 \pm 2.3$ & $61.3 \pm 5.0$ & III \\
\hline Hayamasari & Hokkaido & 1988 & $60.0 \pm 15.1$ & $78.7 \pm 8.1$ & $34.7 \pm 19.2$ & $69.3 \pm 8.1$ & III \\
\hline Himehonami & Hokkaido & 1966 & $47.3 \pm 11.7$ & $68.7 \pm 7.0$ & $34.7 \pm 12.2$ & $60.0 \pm 3.5$ & III \\
\hline Kitaake & Hokkaido & 1983 & $53.3 \pm 2.3$ & $77.3 \pm 7.0$ & $34.7 \pm 4.6$ & $72.7 \pm 8.1$ & III \\
\hline Nanatsuboshi & Hokkaido & 2001 & $50.7 \pm 11.0$ & $77.3 \pm 11.5$ & $34.0 \pm 9.2$ & $64.7 \pm 4.2$ & III \\
\hline Nourin No.20 & Hokkaido & 1941 & $66.0 \pm 10.0$ & $70.7 \pm 1.2$ & $33.3 \pm 8.1$ & $58.7 \pm 4.2$ & III \\
\hline Eiko & Hokkaido & 1942 & $44.7 \pm 4.2$ & $69.3 \pm 11.7$ & $30.0 \pm 5.3$ & $56.7 \pm 15.3$ & III \\
\hline Shiokari & Hokkaido & 1963 & $44.0 \pm 3.5$ & $74.7 \pm 8.1$ & $29.3 \pm 7.6$ & $63.3 \pm 9.0$ & III \\
\hline Hoshimaru & Hokkaido & 2006 & $42.7 \pm 3.1$ & $63.0 \pm 7.1$ & $29.3 \pm 4.2$ & $55.3 \pm 7.6$ & III \\
\hline Shinsetsu & Hokkaido & 1954 & $50.7 \pm 5.0$ & $73.3 \pm 3.1$ & $29.3 \pm 8.1$ & $62.0 \pm 3.5$ & III \\
\hline Ishikari & Hokkaido & 1971 & $52.0 \pm 18.3$ & $60.0 \pm 6.0$ & $26.0 \pm 6.9$ & $41.7 \pm 13.1$ & III \\
\hline
\end{tabular}

Data represent mean \pm SD of triplicates. The evaluation was performed in 2010 .

Breeding varieties are shown with their year of registration. 
To identify QTLs controlling SES, we used 3 mapping populations of BILs using Italica Livorno and Arroz Da Terra as the donor parents and Hayamasari and Hoshinoyume as the recipient parents. Three populations of BILs were developed by the single-seed descent (SSD) method using the procedure described by Fujino et al. (2004). In brief, the $\mathrm{F}_{1}$ plants between the recipient and donor parents were backcrossed with the recipient to produce $\mathrm{BC}_{1} \mathrm{~F}_{1}$ seeds. BILs developed from the resultant $\mathrm{BC}_{1} \mathrm{~F}_{1}$ plants by the SSD method. The HyI population, $\mathrm{BC}_{1} \mathrm{~F}_{6}$, was derived from the cross between Hayamasari showing weak SES and Italica Livorno that exhibits vigorous SES, Hayamasari/Italica Livorno// Hayamasari, and consisted of 122 lines (Fujino et al. 2004). The HsA population, $\mathrm{BC}_{1} \mathrm{~F}_{6}$, was derived from the cross between Hoshinoyume showing weak SES and Arroz Da Terra with vigorous SES, Hoshinoyume/Arroz Da Terra// Hoshinoyume, and consisted of 89 lines (Fujino and Sekiguchi 2005). The HsI population, $\mathrm{BC}_{1} \mathrm{~F}_{6}$, was derived from the cross between Hoshinoyume and Italica Livorno, Hoshinoyume/Italica Livorno//Hoshinoyume, and consisted of 96 lines, which was developed in this study. In 2007 and 2008 , these populations and their parents were cultivated in a paddy field at HOKUREN Agricultural Research Institute (Naganuma, Hokkaido, Japan). Because each BIL showed large variation in heading date (Fujino and Sekiguchi 2005), only lines that reached seed maturity were used for SES evaluation, with 82,71 , and 83 lines in the HyI, HsA, and HsI populations, respectively. All seeds were stored at room temperature until use.

\section{Evaluation of SES}

Evaluation of SES was performed in 2008, 2009, and 2010 in a field at Kamikawa Agricultural Experimental Station (Pippu, Hokkaido, Japan). This field evaluation system is used in the current breeding programs at Kamikawa Agricultural Experimental Station (Fig. 1). Fifty seeds of each line were subjected to the experiment. Seeds were wrapped by seedertape at 10 seeds per $10 \mathrm{~cm}$ (Fig. 1A). The seeds were sown in experimental trays $(30 \mathrm{~cm} \times 60 \mathrm{~cm})$ filled with paddy-field soil to a depth of $1 \mathrm{~cm}$ in early May (Fig. 1B). Then, the tray was placed in a paddy field (Fig. 1C). After setting the tray, irrigation water was allowed to flood the field for the period of the experiment. For the evaluation of SES, seeds with or without pre-germination treatment were used to elucidate the effect of pre-germination treatment on SES, denoted hereafter as G- and NG-, respectively. Firstly, seeds were soaked in water at $11^{\circ} \mathrm{C}$ for 6 days for all experiments. Then, for pre-germination treatment, seeds were placed in an incubator at $28^{\circ} \mathrm{C}$ for 14 hours before sowing. To evaluate SES, we measured 2 traits, emergence of seedlings in soil (ESS) and emergence of seedlings from water (ESW), because growth stages of individuals varied from

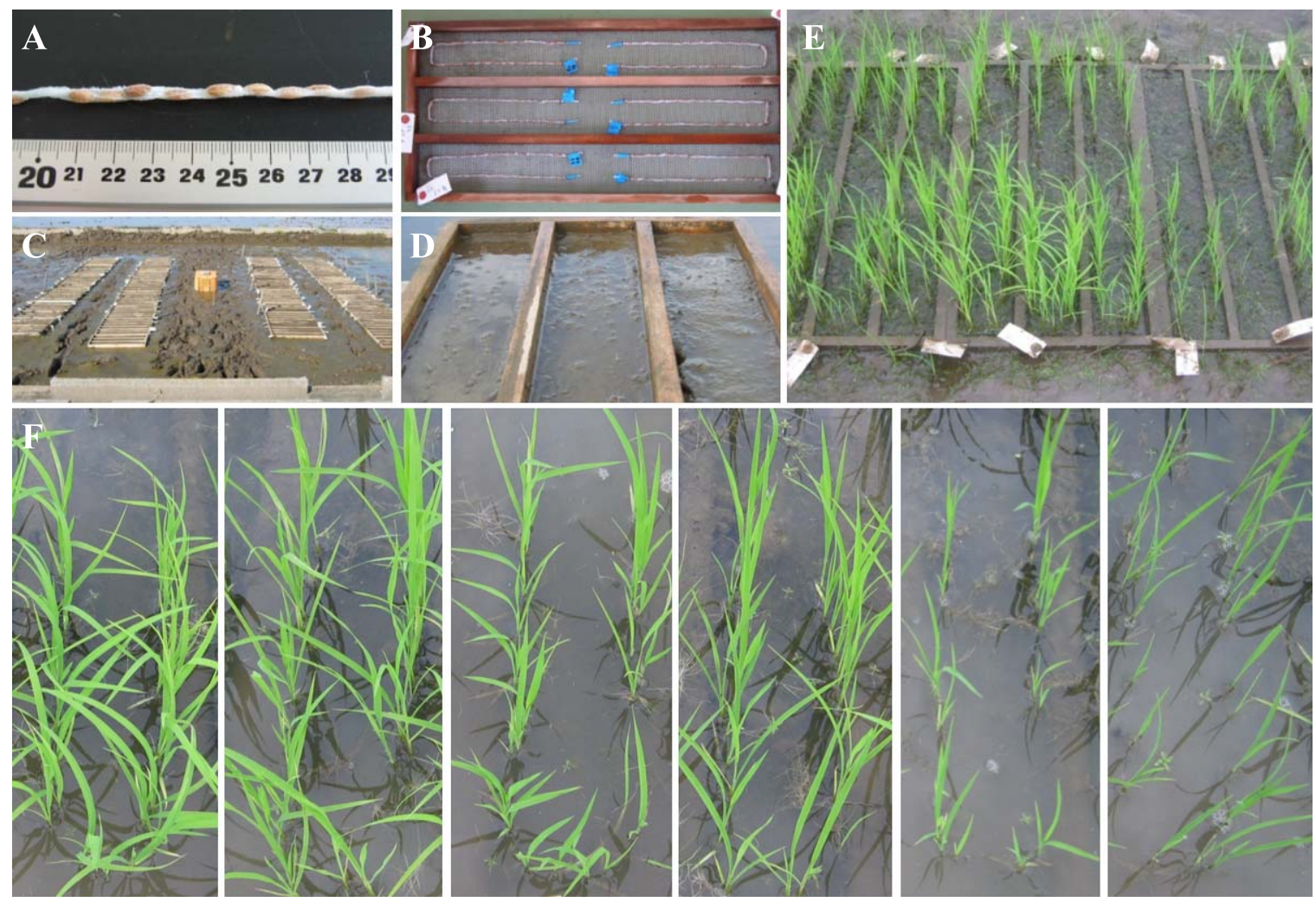

Fig. 1. Photograph of seedling establishment in the field evaluation system. (A) Seeds were wrapped by seeder tape at 10 seeds per $10 \mathrm{~cm}$. (B) The seeds were sown in experimental trays. (C) The tray was placed in a paddy field. (D) Ten and (E) 30 days after sowing. (F) Phenotype of seedling in each SES group. Left to right: Arroz Da Terra, Italica Livorno, Akage, Iburiwase, Hoshinoyume, and Hayamasari. 
2-leaf to 3-leaf seedlings in the evaluation. The degree of tolerance for ESS was measured on the basis of the number of plants for which second leaves emerged from the soil surface. The degree of tolerance for ESW was measured on the basis of the number of plants where third leaves emerged above the flood water surface. Thirty days after sowing, a total of 4 traits were measured for SES: G-ESS, G-ESW, NG-ESS, and NG-ESW (Fig. 1E). The evaluation of varietal difference among rice varieties from Hokkaido was performed in 2010. The evaluations of SES in the HyI, HsA, and HsI populations were performed in 2008/2009, 2008/ 2009 , and 2009, respectively.

\section{DNA analysis}

Total DNA was extracted from the leaves of the BILs and parental lines using the CTAB method with modifications (Murry and Thompson 1980). PCR amplification, gel electrophoresis, and detection were performed in accordance with methods described by Fujino et al. (2004). A linkage map of the HyI population was constructed previously using 139 RFLP and 47 SSR markers (Fujino et al. 2004). Those of the HsA and HsI populations were constructed during the present study using 131 and 129 SSR markers, respectively, distributed over the whole rice genome (Supplemental Fig. 1). Linkage analyses were performed with MAPMAKER/EXP using the Kosambi function (Kosambi 1944, Lander et al. 1987).

\section{QTL analysis}

Detection of QTLs was conducted by composite interval mapping with QTL Cartographer 2.5 (Wang et al. 2010). The threshold to detect QTLs was determined by 1000 permutation tests at a probability level of 0.05 . To normalize the variance, the percentage rate of ESS and ESW was arc-sinetransformed and used for QTL analysis. In this study, QTLs for SES located within the $10 \mathrm{Mb}$ chromosomal region were treated for the same QTL because of the resolution potential by QTL analysis using the primary mapping population.

\section{Results}

Varietal differences in SES among varieties from Hokkaido

Large variations in SES were detected among the varieties tested (Fig. 1F and Table 1). The mean value of NG-ESS was $57.5 \%$, ranging from $44.0 \%$ to $84.7 \%$, while the mean value of NG-ESW was $41.3 \%$, ranging from $26.0 \%$ to $72.0 \%$. The values of G-traits were higher than those of NGtraits, suggesting that pre-germination treatment would enhance SES. On the basis of the phenotypic values of each trait, correlations among traits, and the pedigree of each variety, the degree of SES in the varieties was divided into 3 groups, I, II and III (Table 1). Group I showed vigorous SES and included 3 varieties from Europe. Group II showed intermediate SES and included 4 varieties. Group III showed weak SES and included 17 varieties from the current breeding programs. On the basis of these results, we developed 3 mapping populations of BILs using Arroz Da Terra and
Italica Livorno in group I as donor parents.

\section{Phenotypic variations of ESS and ESW in the 3 mapping populations}

Continuous variations were observed in 4 traits in each mapping population (Table 2, Fig. 2 and Supplemental Fig. 2). The phenotypic variations in 4 traits in the HsA population in 2008 are shown in Fig. 2A. G-ESS and G-ESW of Arroz Da Terra were $77 \%$ and $73 \%$, respectively, which were higher than those of Hoshinoyume at $27 \%$ and $12 \%$, respectively. G-ESS and G-ESW of the BILs varied continuously from $6 \%$ to $66 \%$ and from $2 \%$ to $54 \%$, respectively. NG-ESS and NG-ESW of the BILs showed similar variation patterns to G-ESS and G-ESW. Different variation patterns were detected in the HsI population in 2009 (Fig. 2B). GESS and G-ESW of Italica Livorno were $67 \%$ and $61 \%$, respectively, which were higher than those of Hoshinoyume at $42 \%$ and $32 \%$, respectively. G-ESS and G-ESW of the BILs varied continuously from $8 \%$ to $64 \%$ and from $6 \%$ to $56 \%$, respectively. There were transgressive segregants showing weaker values than Hoshinoyume in all traits. In NG-traits, similar variations were observed with weaker transgressive segregants. Similar variation patterns were also detected in another experiment (Supplemental Fig. 2).

\section{QTLs for SES}

A total of 9 chromosomal regions controlling SES were detected, which explained $10.9 \%$ to $25.6 \%$ of total phenotypic variation (Fig. 3 and Table 3). For all of the QTLs except for $q S E S 5-2$, alleles from the donor parents increased phenotypic values. Among the 9 chromosomal regions, qSES11 on chromosome 11 was the most effective, which explained $25.6 \%$ of total phenotypic variation for NG-SEW in the HsA population. qSES11 also showed higher effects on G-ESS and NG-ESS in the HsA population and on NGSES in the HyI population.

qSES1 on chromosome 1 explained $23.0 \%$ of the total phenotypic variation for G-SES in the HsA population. qSES 2 on chromosome 2 was identified for NG-SEW in the HsA and HyI populations, and explained 19.3\% and 18.1\% of the total phenotypic variation, respectively. Two QTLs, qSES5-1 and qSES5-2, were detected on chromosome 5 because the peaks of the LOD curves were more than $13 \mathrm{Mb}$ apart and the LOD curves were independent. qSES5-1 explained $13.0 \%$ to $16.6 \%$ of the total phenotypic variation. qSES5-2 explained $13.8 \%$ of the total phenotypic variation for NG-ESS in the HsA population. qSES6 on chromosome 6 explained $19.3 \%$ of the total phenotypic variation for GSEW in the HsA population. Two QTLs, qSES7-1 and qSES7-2, were detected on chromosome 7 on the basis of LOD curves. qSES7-1 explained $15.3 \%$ of the total phenotypic variance for G-ESW, and qSES7-2 explained 10.9\% for G-SEW and $17.5 \%$ for G-ESS of the total phenotypic variation in the HsA population. qSES10 on chromosome 10 explained $20.6 \%$ of the total phenotypic variance for GSEW in the HsI population. 
Table 2. Phenotypic value of 3 mapping populations and their parents in the evaluations in 2008 and 2009

\begin{tabular}{|c|c|c|c|c|c|c|c|}
\hline \multirow{2}{*}{ Year } & \multirow{2}{*}{ Population } & \multirow{2}{*}{ Trait } & \multicolumn{2}{|c|}{ Parent (\%) } & \multicolumn{3}{|c|}{ Population (\%) } \\
\hline & & & Donor & Recipient & Maximum & Minimum & Mean \\
\hline \multirow[t]{8}{*}{2008} & HsA & G-ESS & 79.5 & 24.5 & 66.0 & 6.0 & 36.6 \\
\hline & & NG-ESS & 71.0 & 7.5 & 54.0 & 2.0 & 26.3 \\
\hline & & G-ESW & 71.5 & 15.0 & 54.0 & 2.0 & 21.3 \\
\hline & & NG-ESW & 58.5 & 6.0 & 44.0 & 0.0 & 14.9 \\
\hline & HyI & G-ESS & 51.0 & 31.0 & 60.0 & 12.0 & 30.8 \\
\hline & & NG-ESS & 48.0 & 29.5 & 66.0 & 0.0 & 30.8 \\
\hline & & G-ESW & 45.0 & 17.0 & 44.0 & 6.0 & 21.9 \\
\hline & & NG-ESW & 43.0 & 13.0 & 50.0 & 0.0 & 21.2 \\
\hline \multirow[t]{12}{*}{2009} & HsA & G-ESS & 86.0 & 42.0 & 86.0 & 16.0 & 46.1 \\
\hline & & NG-ESS & 79.3 & 32.0 & 66.0 & 8.0 & 34.9 \\
\hline & & G-ESW & 82.0 & 32.0 & 76.0 & 12.0 & 37.8 \\
\hline & & NG-ESW & 77.3 & 28.7 & 56.0 & 6.0 & 27.4 \\
\hline & HyI & G-ESS & 67.3 & 60.0 & 76.0 & 14.0 & 44.2 \\
\hline & & NG-ESS & 72.0 & 48.7 & 82.0 & 16.0 & 44.6 \\
\hline & & G-ESW & 60.7 & 40.7 & 70.0 & 12.0 & 37.2 \\
\hline & & NG-ESW & 69.0 & 36.0 & 82.0 & 12.0 & 39.5 \\
\hline & HsI & G-ESS & 67.3 & 42.0 & 64.0 & 8.0 & 40.6 \\
\hline & & NG-ESS & 72.0 & 32.0 & 72.0 & 4.0 & 37.4 \\
\hline & & G-ESW & 60.7 & 32.0 & 56.0 & 6.0 & 29.9 \\
\hline & & NG-ESW & 69.0 & 28.7 & 58.0 & 0.0 & 31.6 \\
\hline
\end{tabular}

Populations of HsA, HyI, and HsI indicate the crosses between Hoshinoyume and Arroz Da Terra, Hayamasari and Italica Livorno, and Hoshinoyume and Italica Livorno, respectively.
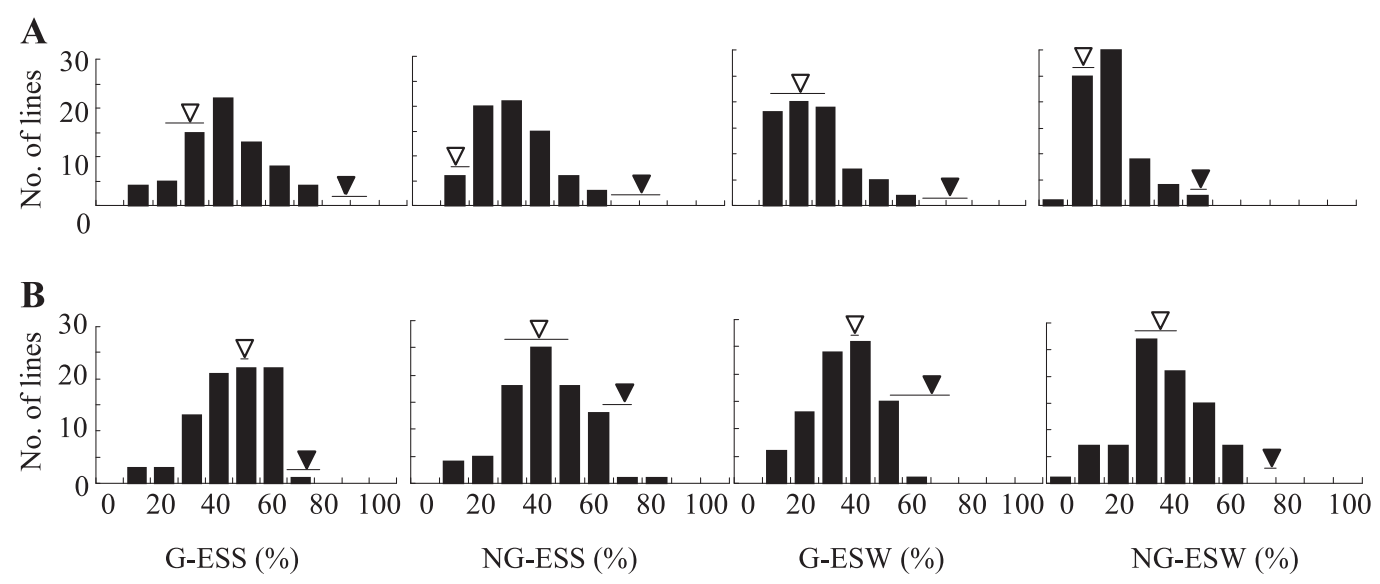

Fig. 2. Frequency distributions of emergence of seedlings in soil (ESS) and emergence of seedlings from water (ESW). (A) HsA population $(n=71)$ in the evaluation in 2008; (B) HsI population $(n=83)$ in the evaluation in 2009. Closed and open arrowheads indicate the mean values for the donor parents and Hoshinoyume, respectively, with ranges (bar).

\section{Discussion}

During the seedling stage of rice, low-temperature causes poor and slow germination, resulting in reduced seedling establishment, non-uniform growth, and reduced yield. Stable SES under natural environmental conditions is an essential trait for rice varieties used for direct seedling methods, especially in local regions with low-temperature. In spite of the significance of tolerance to low-temperature during the seedling stage in direct seeding methods, understanding of the genetic basis of these traits is limited. In Hokkaido, low- temperature in spring causes seedling mortality in direct seeding methods. Not only low-temperature but also other environmental factors cause decreases in SES. In this study, SES was examined by the field evaluation system used in rice breeding programs for continuous growth from seed germination to seedling development. Using Arroz Da Terra and Italica Livorno as the donor parents, a total of 9 chromosomal regions controlling SES have been identified. These QTLs are useful for the improvement of SES in rice breeding programs in local regions with low-temperature, such as Hokkaido. 


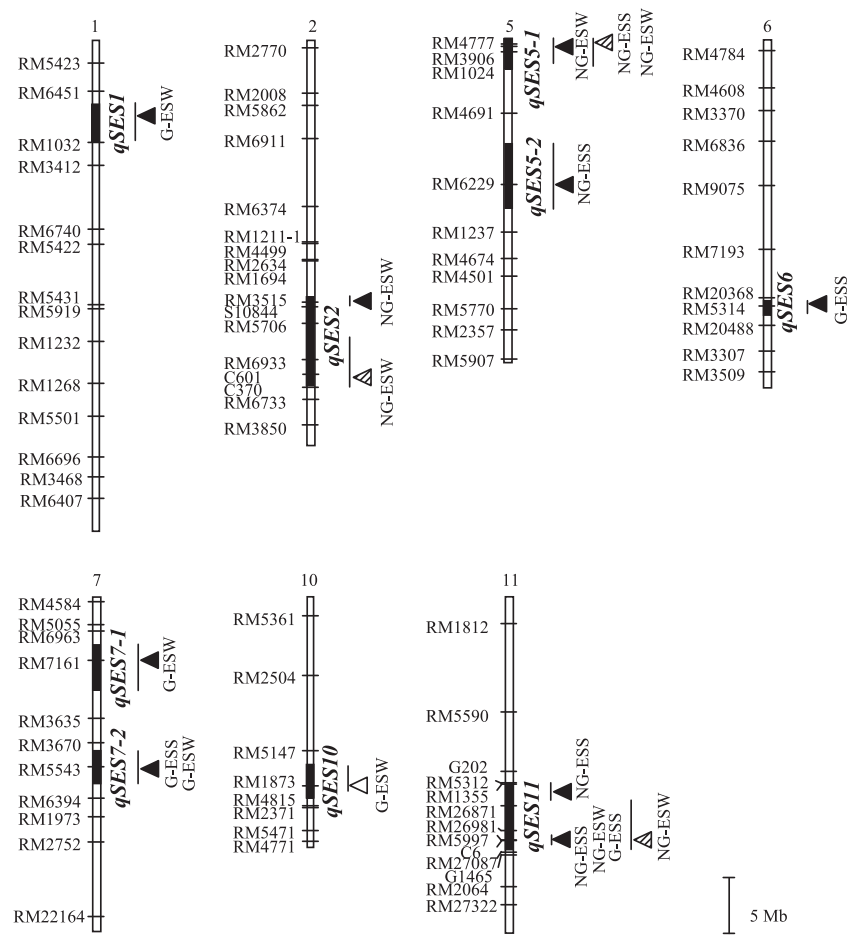

Fig. 3. Chromosomal locations of QTLs for seedling establishment (SES). Closed, open, and hatched triangles indicate the positions of the LOD peaks detected in the HsA, HsI, and HyI populations, respectively. Bars indicate the interval with a decrease of 0.5 from the peak LOD value. Black boxes on the chromosome indicate the chromosomal regions of QTLs for SES. The mapped markers were positioned using pseudo-molecule 3.0 in RAP-DB.
SES was evaluated by the field system used in the current rice breeding programs in this study. QTLs for SES identified in this study will be useful for the introduction of highlevel SES QTLs by marker-assisted selection (MAS) in rice breeding programs. However, most of these QTLs were detected only in a single year and a single population. The results strongly suggested that the expression of these QTLs was sensitive to environmental conditions during the evaluation of SES and the seed maturing stage. It was also unclear which trait is the most important for SES. In this study, we evaluated the 4 traits as indicators of SES. Because SES is a highly complex trait, it is quite difficult to elucidate the relationships between gene (QTL) effects and the evaluation conditions. More comprehensive characterizations of these QTLs identified in this study will be necessary to determine the genetic basis of each QTL, the interactions between these QTLs, and the response to environmental conditions. Previously, we identified QTLs controlling low-temperature germinability (Fujino et al. 2004). A near isogenic line for a major QTL, qLTG3-1, clearly showed more vigorous lowtemperature germinability than the recurrent parent (Fujino et al. 2008, Iwata and Fujino 2010). SES is involved in the continuous growth from seed germination to seedling development. Combining these QTLs may enable establishment of stable SES.

Many QTLs for tolerance to low-temperature in seed germination and seedling stages have been identified using various kinds of mapping populations and various evaluation methods (Andaya and Mackill 2003a, Baruah et al. 2009, Fujino et al. 2004, Ji et al. 2009, Jiang et al. 2006, 2008, Lou et al. 2007, Miura et al. 2001, Zhang et al. 2005a, 2005b). To identify QTLs for tolerance to low-temperature

Table3. QTLs for seedling establishment (SES) in the 3 mapping populations using Italica Livorno and Arroz Da Terra as the donor parents

\begin{tabular}{|c|c|c|c|c|c|c|c|c|c|c|}
\hline \multirow{2}{*}{ Population } & \multirow{2}{*}{ Traits } & \multirow{2}{*}{ QTL } & \multirow{2}{*}{ Marker interval } & \multirow{2}{*}{ Chromosome } & \multicolumn{3}{|c|}{2008} & \multicolumn{3}{|c|}{2009} \\
\hline & & & & & LOD & $\mathrm{AE}^{a}$ & $\mathrm{PVE}^{b}$ & LOD & $\mathrm{AE}^{a}$ & $\mathrm{PVE}^{b}$ \\
\hline \multirow[t]{12}{*}{ HsA } & G-ESS & qSES6 & RM20368-RM5314* & 6 & 4.9 & 4.2 & 19.3 & & & \\
\hline & & qSES7-2 & RM3670-RM5543* & 7 & 3.1 & 3.3 & 10.9 & & & \\
\hline & & qSES11 & RM26981-RM5997* & 11 & 5.1 & 4.8 & 17.2 & & & \\
\hline & NG-ESS & $q S E S 5-2$ & RM4691-RM6229* & 5 & 3.6 & -3.7 & 13.8 & & & \\
\hline & & qSES11 & RM26981-RM5997* & 11 & 5.6 & 4.8 & 20.5 & & & \\
\hline & & qSES11 & RM1355*-RM26871 & 11 & & & & 3.4 & 4.2 & 19.3 \\
\hline & G-ESW & qSES1 & RM6451*-RM1032 & 1 & 3.9 & 5.5 & 23.0 & & & \\
\hline & & qSES7-2 & RM5543*-RM6394 & 7 & 3.6 & 4.5 & 17.5 & & & \\
\hline & & qSES7-1 & RM7161*-RM3635 & 7 & & & & 3.5 & 4.1 & 15.3 \\
\hline & NG-ESW & $q S E S 2$ & RM3515*-RM5706 & 2 & & & & 2.9 & 3.2 & 12.4 \\
\hline & & qSES5-1 & RM4777*-RM1024 & 5 & & & & 2.9 & 2.8 & 13.0 \\
\hline & & qSES11 & RM26981-RM5997* & 11 & 6.2 & 5.2 & 25.6 & & & \\
\hline \multirow[t]{4}{*}{ HyI } & NG-ESS & qSES5-1 & RM4777*-RM3906 & 5 & & & & 4.4 & 7.1 & 16.6 \\
\hline & NG-ESW & $q S E S 2$ & $\mathrm{C} 601 *-\mathrm{C} 370$ & 2 & 4.5 & 4.0 & 18.1 & & & \\
\hline & & qSES5-1 & RM4777*-RM3906 & 5 & & & & 4.0 & 6.3 & 14.1 \\
\hline & & qSES11 & G202-C6* & 11 & & & & 3.6 & 5.1 & 13.9 \\
\hline HsI & G-ESW & qSES10 & RM5147-RM1873* & 10 & & & & 4.7 & 5.0 & 20.5 \\
\hline
\end{tabular}

${ }^{a}$ Additive effect of alleles from donor parents based on arc-sine-transformation of phenotypic rate.

${ }^{b}$ Percentage of total phenotypic variance explained by the QTL.

* Nearest marker to the QTL. 
at the seedling stage, seedlings in various developmental stages grown under optimum conditions were subjected to low-temperature (Andaya and Mackill 2003a, Baruah et al. 2009, Jiang et al. 2008, Lou et al. 2007, Zhang et al. 2005b). In contrast, SES in this study was evaluated as the growth from seed germination to seedling development at continuous low-temperature. Comparison of the chromosomal locations of these QTLs with 9 QTLs for SES identified in this study could help to elucidate the relationships between them as the genetic basis of tolerance to low-temperature in rice. Several QTLs were co-located on a chromosomal region of the most effective locus qSES11. qLTG-11 (Miura et al. 2001), qLTG-11-1, and qLTG-11-2 (Jiang et al. 2006) were identified in the chromosomal region of $q S E S 11$, which controlled seed germination at a temperature of $15^{\circ} \mathrm{C}$. qSCT-11 (Zhang et al. 2005b), qCTS11 and qCTP11 (Baruah et al. 2009), and qCTS11-2 (Andaya and Mackill 2003a) were identified, which controlled response to low-temperature in different developmental stages.

As with the chromosomal region of qSES11, QTLs for tolerance to low-temperature have been identified on the same chromosomal regions as other QTLs for SES identified in this study. $q G R-2$ on chromosome 2 ( $\mathrm{Ji}$ et al. 2009) for qSES2, qLTG-5 (Miura et al. 2001) and qLTG-5-1 (Jiang et al. 2006) on chromosome 5 for $q S E S 5-1, q L T G-5-2$ and $q L T G-5-3$ on chromosome 5 (Jiang et al. 2006) for qSES5-2, qLTG-7 (Jiang et al. 2006) on chromosome 7 for qSES7-1, and $q L T G-10$ on chromosome 10 (Jiang et al. 2006) for qSES10 were identified previously as having roles in seed germination at low-temperature. In addition, $q C T S-1-a$ on chromosome 1 (Lou et al. 2007) for qSES1, qCTS5 on chromosome 5 (Baruah et al. 2009) for qSES5-1, qSCT-5 on chromosome 5 (Jiang et al. 2008) for qSES5-2, qSCT-6 (Jiang et al. 2008) and qCTS6-2 (Andaya and Mackill 2003a) on chromosome 6 for qSES6, and qCTS10 (Andaya and Mackill 2003a) on chromosome 10 for qSES10 were identified previously as controlling responses to lowtemperature in different developmental stages. Only qSES72 from Arroz Da Terra identified in this study appeared to be a novel QTL.

Due to the lack of precise chromosomal locations for these QTLs, the genetic relationships between these QTLs are still unclear as to whether they represent the pleiotropic effect of a single QTL or whether different QTLs are tightly linked to each other. SES in field conditions of Hokkaido involves various kinds of environmental conditions, such as the temperature of soil and flood water, soil quality, and anaerobic conditions caused by flood. The relationships between these QTLs suggested that tolerance to low-temperature is highly important for stable SES in Hokkaido.

Substantial efforts in rice breeding programs for the improvement of tolerance to low-temperature have made rice production stable under natural environmental conditions in Hokkaido. Because of intensive breeding efforts, the genetic diversity of populations in local regions has been reduced (Cuevas-Perez et al. 1992, Dilday 1990), especially in vari- eties from Japan (Yamamoto et al. 2010). The small variation of SES among varieties from Hokkaido identified in this study might be maximized to their genetic potential. QTLs for SES from European varieties identified in this study could be useful in rice breeding programs in Hokkaido. However, it is difficult to introduce vigorous SES from exotic germplasm into commercial rice cultivars due to the large amount of effort required for the elimination of undesirable traits or genes from the germplasm. Both Italica Livorno and Arroz Da Terra have many undesirable traits for rice cultivars in Hokkaido, such as poor straw strength, maturity, shattering, and poor grain quality and shape. The genetic relationships between QTLs for undesirable traits and SES identified in this study could facilitate the utilization of QTLs for SES from this exotic germplasm.

\section{Acknowledgement}

This work was supported in part by a grant from the Ministry of Agriculture, Forestry and Fisheries of Japan (Genomics for Agricultural Innovation QTL-1005).

\section{Literature Cited}

Andaya,V.C. and D.J.Mackill (2003a) Mapping of QTLs associated with cold tolerance during the vegetative stage in rice. J. Exp. Bot. 54: 2579-2585.

Andaya,V.C. and D.J.Mackill (2003b) QTLs conferring cold tolerance at the booting stage of rice using recombinant inbred lines from a japonica $\times$ indica cross. Theor. Appl. Genet. 106: 1084-1090.

Baruah, A.R., N.Ishigo-Oka, M.Adachi, Y.Oguma, Y.Tokizono, K. Onishi and Y.Sano (2009) Cold tolerance at the early growth stage in wild and cultivated rice. Euphytica 165: 459-470.

Cuevas-Perez,F.E., E.P.Guimaraes, L.E. Berrio and D.I.Gonzalez (1992) Genetic base of irrigated rice in Latin America and the Caribbean, 1971 to 1989 . Crop Sci. 32: 1054-1059.

Dai, L., X.Lin, C.Ye, K.Ise, K.Saito, A.Kato, F.Xu, T.Yu and D.Zhang (2004) Identification of quantitative trait loci controlling cold tolerance at the reproductive stage in Yunnan landrace of rice, Kunmingxiaobaigu. Breed. Sci. 54: 253-258.

Dilday, R. (1990) Contribution of ancestral lines in the development of new cultivars of rice. Crop Sci. 30: 905-911.

Fujino, K., H.Sekiguchi, T.Sato, H.Kiuchi, Y.Nonoue, Y.Takeuchi, T.Ando, S.Y.Lin and M.Yano (2004) Mapping of quantitative trait loci controlling low-temperature germinability in rice (Oryza sativa L.). Theor. Appl. Genet. 108: 794-799.

Fujino,K. and H.Sekiguchi (2005) Identification of QTLs conferring genetic variation for heading date among rice varieties at the northern-limit of rice cultivation. Breed. Sci. 55: 141-146.

Fujino, K., H.Sekiguchi, Y.Matsuda, K. Sugimoto, K. Ono and M.Yano (2008) Molecular identification of a major quantitative trait locus, $q L T G 3-1$, controlling low-temperature germinability in rice. Proc. Natl. Acad. Sci. USA 105: 12623-12628.

Iwata, N. and K. Fujino (2010) Genetic effects of major QTLs controlling low-temperature germinability in different genetic backgrounds in rice (Oryza sativa L.). Genome 53: 763-768.

Ji,S.L., L.Jiang, Y.H.Wang, W.W.Zhang, X.Liu, S.J.Liu, L.M.Chen, H.Q.Zhai and J.M.Wan (2009) Quantitative trait loci mapping and stability for low temperature germination ability of rice. Plant 
Breed. 128: 387-392.

Jiang,L., S.Liu, M.Hou, J.Tang, L.Chen, H.Zhai and J.Wan (2006) Analysis of QTLs for seed low temperature germinability and anoxia germinability in rice (Oryza sativa L.). Field Crops Res. 98: 68-75.

Jiang,L., M.Xun, J.Wang and J.Wan (2008) QTL analysis of cold tolerance at seedling stage in rice (Oryza sativa L.) using recombination inbred lines. J. Cereal Sci. 48: 173-179.

Kosambi,D.D. (1944) The estimation of map distances from recombination value. Ann. Eugen. 12: 172-175.

Kuroki,M., K.Saito, S.Matsuba, N.Yokogami, H.Shimizu, I.Ando and Y.Sato (2007) A quantitative trait locus for cold tolerance at the booting stage on rice chromosome 8. Theor. Appl. Genet. 115: 593-600.

Lander,E.S., P.Green, J.Abrahamson, A. Barlow, M.J.Daly, S.E. Lincoln and L.Newburg (1987) MAPMAKER: an interactive computer package for constructing primary genetic linkage maps of experimental and natural populations. Genomics 1: 174-181.

Lou, Q., L.Chen, Z.Sun, Y.Xing, J.Li, X.Xu, H.Mei and L.Luo (2007) A major QTL associated with cold tolerance at seedling stage in rice (Oryza sativa L.). Euphytica 158: 87-94.

Miura,K., S.Y.Lin, M.Yano and T.Nagamine (2001) Mapping quantitative trait loci controlling low temperature germinability in rice (Oryza sativa L.). Breed. Sci. 51: 293-299.

Murray,M.G. and W.F.Thompson (1980) Rapid isolation of high molecular weight plant DNA. Nucleic Acids Res. 8: 4321-4326.

Nakagahra, M., K.Okuno and D.Vaughan (1997) Rice Genetic resources: history, conservation, investigative characterization and use in Japan. Plant Mol. Biol. 35: 69-77.

Peterson,M.L., D.B.Jones and J.N.Rutger (1978) Cool temperature screening of rice lines for seedling vigor. II RISO 27: 269-274.

Saito,K., K.Miura, K.Nagano, Y.Hayano-Saito, A.Saito, H.Araki and
A. Kato (1995) Chromosomal location of quantitative trait loci for cool tolerance at the booting stage in rice variety 'Norin-PL8'. Breed. Sci. 45: 337-340.

Suh,J.P., J.U.Jeung, J.I.Lee, Y.H.Choi, J.D.Yea, P.S.Virk, D.J.Mackill and K.K.Jena (2010) Identification and analysis of QTLs controlling cold tolerance at the reproductive stage and validation of effective QTLs in cold-tolerant genotypes of rice (Oryza sativa L.). Theor. Appl. Genet. 120: 985-995.

Takeuchi, Y., H. Hayasaka, B.Chiba， I. Tanaka， T. Shimano, M. Yamagishi, K.Nagano, T.Sasaki and M.Yano (2001) Mapping quantitative trait loci controlling cool-temperature tolerance at booting stage in temperate japonica rice. Breed. Sci. 51: 191-197.

Wang, S., C.J.Basten and Z.-B.Zeng (2010) Windows QTL Cartographer 2.5. Department of Statistics, North Carolina State University, Raleigh, North Carolina, USA. http://statgen.ncsu.edu/qtlcart/ WQTLCart.htm

Yamamoto, T., H.Nagasaki, J.Yonemaru, K.Ebana, M.Nakajima, T.Shibaya and M.Yano (2010) Fine definition of the pedigree haplotypes of closely related rice cultivars by means of genomewide discovery of single-nucleotide polymorphisms. BMC Genomics 11: 267 doi: 10.1186/1471-2164-11-267.

Zhang,Z.H., X.S.Qu, S.Wan, L.H.Chen and Y.G.Zhu (2005a) Comparison of QTL controlling seedling vigour under different temperature conditions using recombinant inbred lines in rice (Oryza sativa). Ann. Bot. 95: 423-429.

Zhang,Z.H., L.Su, W.Li, W.Chen and Y.G.Zhu (2005b) A major QTL conferring cold tolerance at the early seedling stage using recombinant inbred lines of rice (Oryza sativa L.). Plant Sci. 168: 527-534.

Zhou,L., Y.Zeng, W.Zheng, B.Tang, S.Yang, H.Zhang, J.Li and Z.Li (2010) Fine mapping a QTL $q C T B 7$ for cold tolerance at the booting stage on rice chromosome 7 using a near-isogenic line. Theor. Appl. Genet. 121: 895-905. 